\title{
Imprecise probabilities
}

\section{GERT DE COOMAN}

Research Associate of the Fund for Scientific Research, Flanders, Belgium. Universiteit Gent, Onderzoeksgroep Systems, Technologiepark, Zwijnaarde 9, 9052 Jwijnaarde, Belgium. E-mail address: gert.decooman@rug.ac.be

The papers in the present symposium are a selection of contributions presented at ISIPTA '99, the first International Symposium on Imprecise Probabilities and Their Applications, held in Ghent, Belgium, from 29 June to 2 July 1999.

'Imprecise probability' is meant as a generic term for the many mathematical models which measure chance or uncertainty without sharp numerical probabilities. Such models are needed in inference problems where the relevant information is scarce, vague, or conflicting, and in decision problems where preferences are imcomplete. Imprecise probability models are currently being studied and applied by a large number of researchers working in a great variety of fields. The aim of ISIPTA '99 was (i) to bring these people together to present research and to discuss issues of common interest, and (ii) to provide a common meeting place that would enable the various theories of imprecise probabilities that have been developed to be discussed and compared.

More than fifty carefully reviewed papers were presented at ISIPTA '99 (Cooman, Cozman, Moral and Walley, 1999), on a wide range of topics: mathematical models for uncertainty, conditioning rules, models for independence, combination of uncertainties, algorithms for computing inferences, coherence, hierarchical models, imprecise Markov processes, decision theory, ambiguity aversion, and Ellsberg's experiment. There were also papers on applications in economics, decision making, statistical inference, experimental studies of human judgement, artifical intelligence, reliability, dynamical systems, robotics, civil engineering, classification, and legal problems.

In preparing this RDP symposium, I faced the problem of selecting important papers that, taken together, would be representative for the rest of the contributions to ISIPTA '99, in the sense that they would somehow convey the distinct ambiance of open debate, free exchange of ideas and multidisciplinarity that many ISIPTA '99 participants were so enthusiastic about. I believe my final choice to be fairly balanced: you will find two theoretical survey papers, one on independence and one on decision making; two papers on psychological experiments related to interesting problems in imprecise probability theory, namely updating beliefs and sample space ignorance; and an applied mathematical paper dealing with optimal pollution control.

In A survey of concepts of independence for imprecise probabilities, Couso et al. discuss, motivate, and compare various definitions of independence in the context of imprecise probabilities. They argue that, whereas there is essentially only one definition of independence for precise probabilities, there are at least six different notions or aspects of independence when imprecision is allowed, all of which are useful in different contexts. Some may see this as a disadvantage of working with imprecise probabilities. I prefer to think it is an advantage: imprecise models allow us 
to distinguish between several notions that arguably, and to a certain extent, are conflated when enforcing precision.

Levi's overview paper Imprecise and indeterminate probabilities is a discussion of various views on rational decision making. Using a simple example, Levi compares and contrasts the so-called strict Bayesian, quasi-Bayesian, maximalist, and maximin approaches. He defends the quasi-Bayesian position, which holds that beliefs cannot always be represented by precise probabilities, but that admissible actions in a decision problem should always be Bayes actions, in the sense that they should maximize expected utility for some (precise) probability measure and utility function.

In An experimental study of updating ambiguous beliefs, Cohen et al. consider two rules that are often used for conditioning, or updating, imprecise probability models: the maximum likelihood update - also called Dempster's (1967) rule - and the full Bayesian update - also called the natural extension rule (Walley, 1991; Walley and Cooman, 1999). The authors describe an experiment related to Ellsberg's (1961), that they argue is able to test the descriptive validity of the two rules, as they lead to clearly different preferences after updating.

A second experimentally oriented paper is Human judgment under sample space ignorance by Smithson et al., who discuss a number of experiments dealing with how people bet on events under so-called sample space ignorance, i.e., when there is little or no information about what the possible outcomes are. Four guiding principles for betting - or providing upper and lower probabilities - under such circumstances were given by Walley (1991, 1996), who also suggested a model for doing so, and for updating under new sample information, called the Imprecise Dirichlet model (IDM) (1996). The IDM satisfies these principles. The described experiments were designed to test whether people behave according to Walley's prescriptions, and whether their betting is in accordance with the IDM.

In Optimal pollution control under imprecise environmental risk and irreversibility, Chevé and Congar loot at the problem of finding optimal consumption strategies under a number of constraints: they assume that consumption generates pollution, and that pollution, when reaching a certain critical level, may give rise to catastrophic environmental events. The question is then to find strategies that maximize the accumulated net utility (i.e., the difference between consumption utility and pollution disutility) and at the same time avoid environmental catastrophies. In many cases, the critical pollution level above which a catastrophe occurs, is not very well known, and is therefore assumed to be a random variable. But even then, so little may be known about it that it is impossible to specify its distribution function in precise terms. The authors use a particular imprecise probability model, first considered by Dempster (1967), to study this kind of situation. Their work shows that using imprecise probabilities is feasible in practical modelling and control problems. A number of conclusions drawn from their model seem to support cautious and conservative policies with respect to pollution and damage to the environment.

\section{References}

de Cooman, G., Cozman, F.G., Moral, S. and Walley, P. editors. ISIPTA '99 (1999), Proceedings of the First International Symposium on Imprecise Probabilities and Their Applications, Imprecise Probabilities Project, Ghent. 
Dempster, A.P. (1967), 'Upper and lower probabilities generated by a random closed interval', Annals of Mathematical Statistics, 39: 957-966.

Ellsberg, D. (1961), 'Risk, ambiguity and the Savage axioms', Quarterly Journal of Economics, 75: 643-669.

Shafer, G. (1976), A Mathematical Theory of Evidence, Princeton, NJ: Princeton University Press.

Walley, P. (1991), Statistical Reasoning with Imprecise Probabilities, London: Chapman \& Hall.

Walley, P. (1996), 'Inferences from multinomial data: learning about a bag of marbles', Journal of the Royal Statistical Society, Series B, 58: 3-57 (with discussion).

Walley, P. and de Cooman, G. (1999), 'Coherence of rules for defining conditional possibility', International Journal of Approximate Reasoning, 21: 63-107. 